\title{
A novel broad-band neutron spin filter based on dynamically polarized protons using photo-excited triplet states
}

\author{
Yifan Quan ${ }^{\mathrm{a}}$, Nemanja Nekitic ${ }^{\mathrm{b}}$, Ben van den Brandt, and Patrick Hautle \\ Laboratory for Scientific Developments and Novel Materials (LDM), Paul Scherrer Institute, 5232 Villigen PSI, Switzerland
}

\begin{abstract}
The use of polarized protons as a broad-band neutron spin filter is an attractive alternative to the well-established neutron polarization techniques, namely polarized ${ }^{3} \mathrm{He}$ gas and super mirrors, since the spindependent neutron proton scattering cross-section is large in a broad wavelength range. We have developed a novel neutron spin filter where we create the necessary large proton polarization in a solid with a recent method of dynamic nuclear polarization (DNP) that uses photo-excited triplet states. This requires only moderate experimental means and allows a compact design.

In order to quantify the efficiency of the spin filter, we have measured the relevant spin-dependent and spinindependent terms of the neutron scattering cross-section of a naphthalene single crystal. The data allows to estimate the triplet spin filter performance over a broad wavelength range. With the recently achieved proton polarization of $80 \%$ the triplet filter compares well with a state of the $\operatorname{art}^{3} \mathrm{He}$ filter.
\end{abstract}

\section{Introduction}

Polarized neutrons are widely used in fundamental physics studies as well as for material investigations in neutron scattering science and the main polarization techniques, super mirrors and ${ }^{3} \mathrm{He}$ filters have constantly been further developed with considerable effort. However, already in the 1960's, Lushchikov and his colleagues demonstrated that alternatively a polarized proton target can provide an ultimate broad-band neutron spin filter [1]. Due to the technically demanding dynamic nuclear polarization (DNP) process [2], this way has found restricted application only. Recently we demonstrated that using short lived photo-excited triplet states instead of stable radicals for DNP [3], the necessary high proton polarization can be reached in a naphthalene single crystal with only moderate experimental means, i.e at a magnetic field of $\sim 0.4 \mathrm{~T}$ and a temperature between 25 and $100 \mathrm{~K}$ [4]. It has been further demonstrated that the method can be employed to build an efficient neutron spin filter suited as primary polarizer for a white cold neutron beam [5] or as spin analyser for small angle neutron scattering (SANS) probing magnetic interactions [6].

The neutron polarization process for polarized protons is based on scattering and not absorption, as is the case for polarized ${ }^{3} \mathrm{He}$. Thus, it should have advantages at higher neutron energies, provided the relevant polarization cross section is sufficiently large. Particularly, for thermal or even epithermal neutrons, which are also of interest for fundamental physics studies, such as the proposed T-violation search [7], an efficient ${ }^{3} \mathrm{He}$ spin filter would need dimensions and a pressure at the limits of current techniques $[8,9]$.

\footnotetext{
a e-mail: yifan.quan@psi.ch

b Now at: CSEM Neuchâtel, 2002 Neuchâtel, Switzerland
}

In order to better quantify the performance of our triplet spin filter, a sound data set for the relevant neutron proton cross-sections in naphthalene as function of the wavelength is needed. First measurements of the polarization dependent cross section have been presented in [10], while in this note we report on experiments that extend the data set, which is then used to asses the efficiency of the spin filtering process.

\section{Spin-dependence of the neutron-proton scattering}

The working principle of a polarized proton spin filter is based on the fact that the singlet cross section for neutron-proton scattering is much higher than the triplet cross section [11]. Neutrons with spin anti-parallel to the proton polarization will be much stronger scattered, while neutrons with spin parallel to the proton polarization are preferably transmitted. The Bragg cutoff wavelength is about $14 \AA$, above which only incoherent scattering on bound protons takes place and the spin dependent incoherent cross section per nucleus is given by [11]

$$
\sigma_{i n c}=\pi b_{N}^{2}\left[I(I+1)-I^{2} P^{2}-I p P\right]
$$

where $b_{N}=-(5.824 \pm 0.002) \times 10^{-12} \mathrm{~cm}$ is the spindependent scattering length difference [12], $p$ the neutron polarization, $I=\frac{1}{2}$ and $P$ the proton polarization. Putting in numerical values and assuming a fully polarized neutron beam $(p=1)$ we obtain

$$
\sigma_{ \pm}=79.9\left[1-\frac{1}{3} P^{2} \mp \frac{2}{3} P\right] \times 10^{-24} \mathrm{~cm}^{2}
$$

where the \pm stands for the two eigenstates of the neutron spin. In the intermediate energy range between 3 and $0.3 \AA$, the cross sections become very complex and cannot be 
theoretically predicted due to interference and inelastic scattering. We generalize Eq. (2) to the form [1]

$$
\sigma_{ \pm}=\sigma_{0}^{0}-\sigma_{0}^{P} P^{2} \mp \sigma_{p} P
$$

where $\sigma_{0}^{0}, \sigma_{0}^{P}$ and $\sigma_{p}$ are empirical parameters. The neutron spin-independent cross-section includes two terms $\sigma_{0}^{0}$ and $\sigma_{0}^{P}$, where the latter depends on the proton polarization, while $\sigma_{p}$ is the so called "polarization crosssection".

For neutron energies larger than inter-atomic bonds $(E>1 \mathrm{eV}, \quad \lambda<0.3 \AA)$ the cross sections approach those of the isolated free nuclei. Then for protons $\sigma_{P}=16.7$ barn, $\sigma_{0}=20.36$ barn [1] and for carbon nuclei $\sigma_{0}=4.7$ barn [13].

When passing through a spin filter with a proton density $N$ and a thickness $d$, the two spin components of the neutron beam will be attenuated according to

$$
T_{ \pm}=e^{-\sigma_{ \pm} N d}
$$

and an initially unpolarized beam will acquire a polarization

$$
A=\frac{T_{+}-T_{-}}{T_{+}+T_{-}}=\tanh \left(-\sigma_{P} P N d\right)
$$

which is called the filter analyzing power. The total transmission $T$ through the filter increases with its polarization $P$ :

$T=\frac{T_{+}+T_{-}}{2}=\exp \left[-\left(\sigma_{0}^{0}-\sigma_{0}^{P} P^{2}\right) N d\right] \cosh \left(-\sigma_{P} P N d\right)$

The filter quality factor is often judged by its figure of merit

$$
Q=A^{2} T
$$

So far no experimental values for the neutron spinindependent cross sections have been reported for naphthalene in order to reliably evaluate $Q$. This lack has been addressed and is reported in the following.

\section{Cross-sections measurements}

\subsection{Experimental set-up}

The experiment was performed at the BOA beam line [14], at the continuous spallation neutron source SINQ at the Paul Scherrer Institute (PSI) in Switzerland. A schematic view of the experimental set-up is given in Fig. 1. The incident beam is polarized positively and with an adiabatic spin flipper the neutron spin state can be reversed with an efficiency $f$ close to 1 . A guide field perpendicular to the neutron path maintains the neutron polarization up to the spin filter. In order to perform a wavelength dependent measurement, a chopper is placed after the spin flipper and the time of flight (TOF) is determined over the distance of $4.5 \mathrm{~m}$ to a ${ }^{3} \mathrm{He}$ neutron detector.

\subsection{Determination of the spin-dependent cross-section}

To determine the spin-dependent cross-section $\sigma_{p}$ we measured the flipping ratio $R$ [15], which is the ratio of

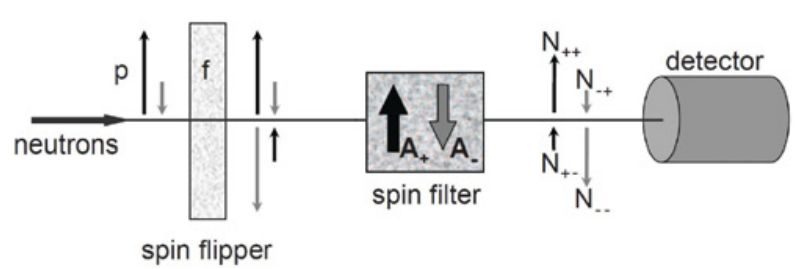

Figure 1. Schematic view of the measurement set-up.

the count rates $N_{--}$with neutron and proton spin parallel and $N_{+-}$antiparallel:

$$
R=\frac{N_{--}}{N+-}=\frac{1+f p A}{1-p A}
$$

where the analysing power $\mathrm{A}$ is given by Eq. (5). The proton polarization $P$ was negative throughout the experiment and the incoming beam was polarized positively $(p>0)$, so the flipper efficiency enters in the numerator. The measurements and data evaluation have been performed as described in detail in [10]. Due to the higher proton polarization, the new data sets have better statistics but are still limited by the precision of the knowledge of the beam polarization, especially below $2 \AA$.

\subsection{Determination of the spin-independent cross-section}

The neutron spin-independent part of the effective cross section (Eq. (3)) consist of two terms $\sigma_{0}$ and $\sigma_{0}^{P} P^{2}$, where the former can be conveniently determined from the transmission through the unpolarized filter according to Eq. (6)

$$
T(P=0)=\frac{I_{\text {spin filter }(P=0)}}{I_{\text {incident beam }}}=e^{-\sigma_{0}^{0} N d}
$$

The result of such a measurement (see Fig. 2) can be checked by comparing the experimental value of the product $\sigma_{0}^{0} d$ to the theoretical scattering cross section given in Eq. (2). The scaling factor represents the thickness of the crystal $d$. The fitted value of the crystal thickness $d_{f}=4.89 \pm 0.06 \mathrm{~mm}$ is in good agreement with the directly measured thickness $d_{m}=4.75 \pm 0.2 \mathrm{~mm}$ and gives a high confidence in the experimental results. The data for $\sigma_{0}^{0}$ shows a pronounced peak at around $6 \AA$, which originates from coherent scattering caused by the specific crystal orientation used in the experiment.

Additionally, by comparing the transmission of an unpolarized neutron beam through a polarized and unpolarized target, respectively, $\sigma_{0}^{P}$ can be determined via

$$
\frac{T(P>0)}{T(P=0)}=e^{-\sigma_{0}^{P} P^{2} N d} \cosh \left(-\sigma_{p} P N d\right)
$$

However, the effect of the spin filter polarization on the transmission is not very strong. Considering the polarization values between 0.40 and 0.60 used in this experiment and according to Eq. (2) $\sigma_{0}^{P}$ has a maximum value of $\frac{1}{3} \sigma_{0}^{0}$ in the long wavelength range, we could expect only few percent modulation of the transmission by the spin filter polarization. It turned out that this was not sufficient to evaluate $\sigma_{0}^{P}$ reliably. 


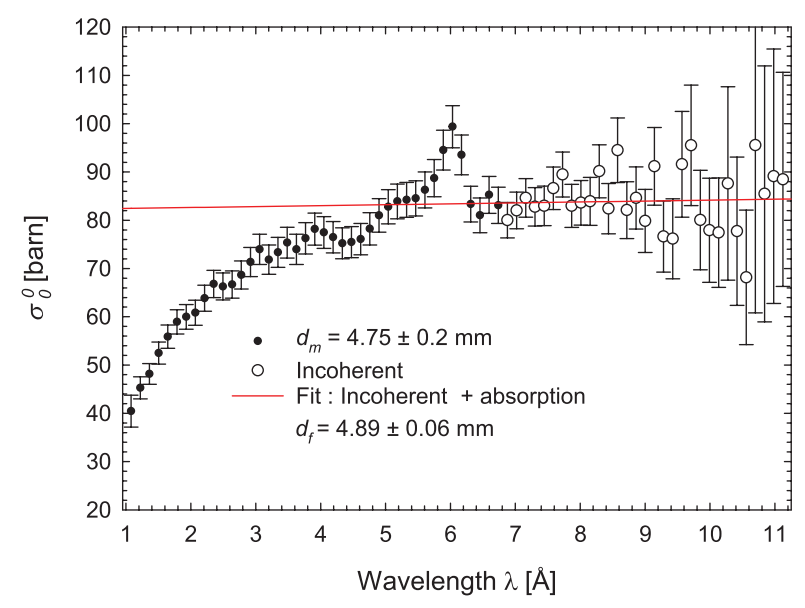

Figure 2. Wavelength dependence of the $\sigma_{0}^{0}$ cross section. The value obtained for the crystal thickness $d$ from fitting the theoretical value to the data above $7 \AA$ is in good agreement with the directly measured value. Notice, the small slope of the fit curve originates from the absorption that has been included [10-12].

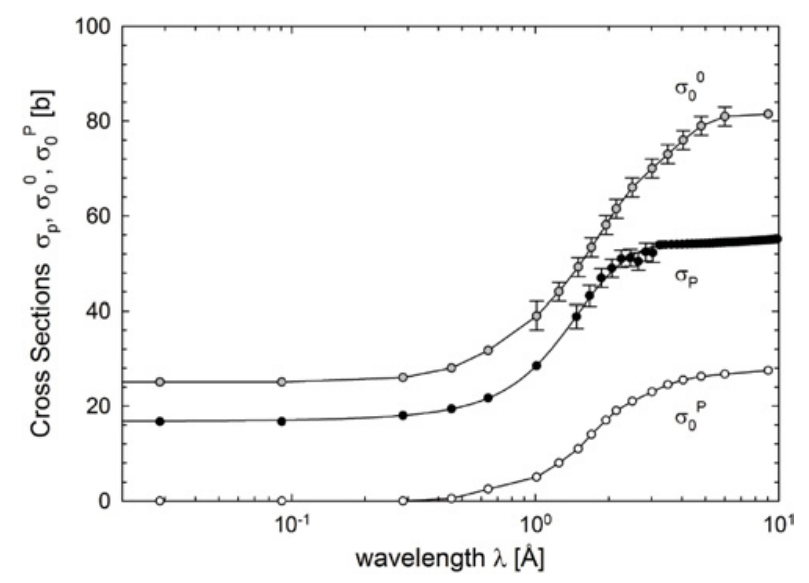

Figure 3. Wavelength dependence of the neutron-proton scattering cross sections $\sigma_{0}^{0}, \sigma_{0}^{P}$ and $\sigma_{p}$ for naphthalene. The values plotted without error bars are adapted from literature (see text). The lines are drawn to guide the eye.

Figure 3 compiles our results for the three crosssections together with values published previously $[1,16]$. For $\sigma_{0}^{0}$ we have smoothed the data, i.e. we have neglected the effect of any Bragg scattering as it depends on the exact orientation of the crystal which we are free to choose. For $\sigma_{0}^{P}$ we give an estimate which is based on the theoretical values for the long neutron wavelength range and on literature values for the short wavelength range [1]. Moreover, below $1 \AA$, the scattering cross-section for carbon has also been included [13].

\section{Spin filter efficiency}

The effective cross-sections at hand, we can now calculate the efficiency of the spin filter, i.e. the transmission $T$, analyzing power $A$ and the figure of merit $Q=A^{2} T$ at a given proton polarization $P$, crystal thickness $d$ and neutron wavelength $\lambda$. Figure 4 shows the results of simulations for two specific wavelengths, $6 \AA$ and $0.33 \AA$, respectively. The former is a typical wavelength used in small angle neutron scattering (SANS) experiments and
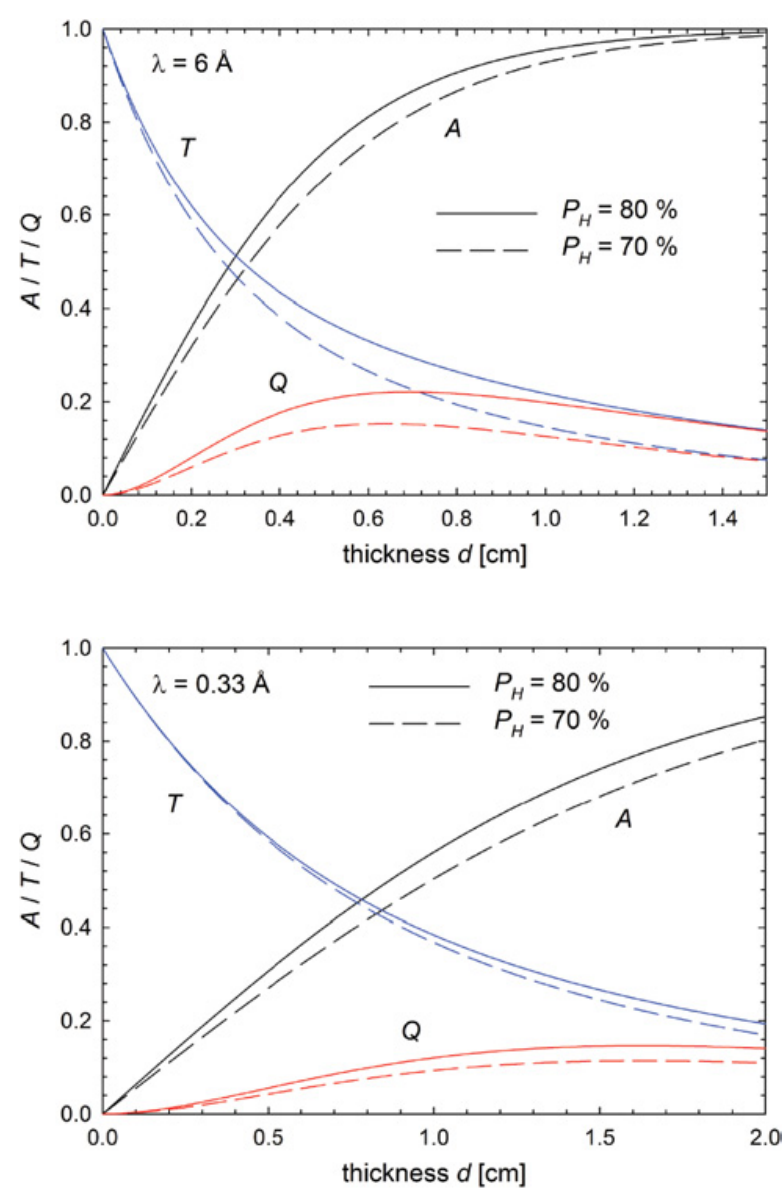

Figure 4. Performance of a naphthalene neutron spin filter based on polarized protons. Plotted are the analyzing (polarizing) efficiency $A$, the total transmission $T$ and the figure of merit $Q$ as a function of the thickness $d$ for two wavelengths, (top): $6 \AA$ [6] and (bottom): $0.33 \AA$.

the latter corresponds to the $0.734 \mathrm{eV} p$-wave resonance in ${ }^{139} \mathrm{La}$ used in time reversal invariance violation searches [7]. In the long wavelength range the optimum thickness is around $7 \mathrm{~mm}$, whereas for epithermal neutrons $\sigma_{p}$ is smaller and thus the optimum filter thickness is larger, around $15 \mathrm{~mm}$.

Furthermore we may plot the figure of merit $Q$ of the triplet filter as a function of the wavelenght and compare it with polarized gaseous ${ }^{3} \mathrm{He}$ (Fig. 5). With the recently achieved proton polarization of 0.8 in a $4.4 \mathrm{~mm}$ thick naphthalene sample [17], the triplet filter gives a $Q$ comparable to that of ${ }^{3} \mathrm{He}$ filter with $P=0.7$, optimized for $3 \AA$. By choosing a specific opacity factor of the ${ }^{3} \mathrm{He}$ gas cell, given by the pressure-lenght-wavelenght product in bar-cm- $\AA$, the $Q$ of the ${ }^{3} \mathrm{He}$ spin filter is optimized for a specific wavelength. In the case of naphthalene, as illustrated in Fig. 5, one either optimizes the filter thickness for cold neutrons $(d \sim 7-8 \mathrm{~mm})$ or for the thermal and epithermal range $(d>10 \mathrm{~mm})$ and obtains a high $Q$ in a broad energy range. The triplet filter might be the preferred way to polarize high energy neutrons, since the opacity factor of the ${ }^{3} \mathrm{He}$ spin filter gets limited by practical restrictions. Notice, an increase in polarization results not only in an improvement of the filter analyzing power $A$ but even more of the transmission $T$. Thus the improvement in 


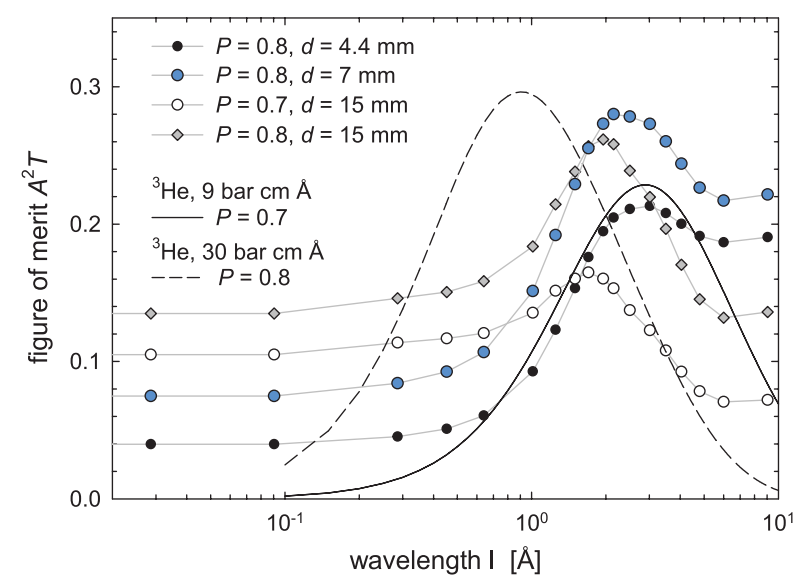

Figure 5. Figure of merit $Q$ of the triplet spin filter for different thicknesses compared to high opacity polarized ${ }^{3} \mathrm{He}$ cells. The plotted values for $Q$ are determined from the data presented in Fig. 3 and the error is estimated to be about $5 \%$. The black dots give the $Q$ of the filter presently used for spin analysis in SANS experiments [17].

polarization from 0.7 to 0.8 has lead to a $40 \%$ gain in the figure of merit compared to previous results [5].

\section{Outlook and conclusion}

Increasing the filter size is the obvious next development step and we are presently testing a modified apparatus capable to polarize up to $1 \mathrm{~cm}^{3}$ crystals. Additionally there is a way to greatly increase the actual filter cross section without having to scale the polarization apparatus up to eventually unmanageable size and complexity: one places the filter in a neutron optics focus and de-focus scheme [5]. Simulations show that such a scheme is also feasible for higher energy neutrons. A set of $20 \mathrm{~m}$ long $m=7$ ballistic supermirror guides would be suited to use a $40 \times 40 \mathrm{~mm}^{2}$ neutron beam of $0.7 \mathrm{eV}$ with a $10 \times 10 \mathrm{~mm}^{2}$ filter. Such a neutron optic system would have the further advantage to provide a much higher neutron intensity with considerably less background, compared to a setup without guides.

In conclusion, we present a complete set of neutron proton cross-sections that allows us to quantify the efficiency of the triplet spin filter over the whole neutron energy range. Using this data set, we find that for the presently achieved level of proton polarization of $P=0.8$, we obtain a figure of merit of more than 0.20 with a $5 \mathrm{~mm}$ thick filter crystal, which is comparable to a $70 \%$ polarized ${ }^{3} \mathrm{He}$ filter. We are presently working on enlarging the filter thickness, which would considerably improve the performance, especially for thermal and epithermal neutrons.
We wish to thank W.Th. Wenckebach for many helpful discussions and U. Filges for the MacStas simulation of the neutron focusing schemes. This work was supported by the Swiss National Science Foundation grants 200021_165496 and 200021_143297.

\section{References}

[1] V.I. Lushchikov, Yu.V. Taran, F.L. Shapiro, Soviet J. Nucl. Phys. 10, 669 (1969)

[2] A. Abragam, M. Goldman, Rep. Prog. Phys. 41, 395 (1978)

[3] A. Henstra, T.S. Lin, J. Schmidt, W. Th. Wenckebach, Chem. Phys. Lett. 165, 6 (1990)

[4] T.R. Eichhorn, M. Haag, B. van den Brandt, P. Hautle, W.Th. Wenckebach, S. Jannin, J.J. Van der Klink, A. Comment, J. Magnetic Resonance 234, 58 (2013)

[5] T.R. Eichhorn, N. Niketic, B. van den Brandt, U. Filges, T. Panzner, E. Rantsiou, W. Th Wenckebach, P. Hautle, Nucl. Instrum. Meth. Phys. Res. Section A 754, 10 (2014)

[6] N. Niketic, B. van den Brandt, W. Th. Wenckebach, J. Kohlbrecher, P. Hautle, J. Appl. Crystallography 48, 1514 (2015)

[7] J.D. Bowman, V. Gudkov, Phys. Rev. C 90, 065503 (2014)

[8] W.C. Chen, T.R. Gentile, R. Erwin, S. Watson, Q. Ye, K.L. Krycka, B.B. Maranville, J. Physics: Conf. Ser. 528, 012014 (2014)

[9] T.R. Gentile, P.J. Nacher, B. Saam, T.G. Walker, Rev. Mod. Phys. 89, 045004 (2017)

[10] M. Haag, B. van den Brandt, T. R. Eichhorn, P. Hautle, W. Th Wenckebach, Nucl. Instrum. Meth. Phys. Res. Section A 678, 91 (2012)

[11] H. Glättli, M. Goldman, Methods in Experimental Physics (Academic Press, New York, 1987), p. 241

[12] A.-J. Dianoux, G. Lander, ILL Neutron Data Booklet (OCP Science Imprint, Philadelphia, 2003)

[13] O. Gritzaya, V. Kolotyi, N. Klimova, O. Kalchenko, M. Gnidak, P. Vorona, Int. Conf. Nucl. Data Sci. Tech. (2007)

[14] M. Morgano, S. Peetermans, E.H. Lehmann, T. Panzner, U. Filges, Nucl. Instrum. Meth. Phys. Res. Section A 754, 46 (2014)

[15] O. Zimmer, Phys. Lett. B 461, 307 (1999)

[16] S. Hiramatsu, S. Isagawa, S. Ishimoto, A. Masaike, K. Morimoto, S Funahashi, Y. Hamaguchi, N. Minakawa, Y. Yamaguchi, J. Phys. Soc. Japan 45, 949 (1978)

[17] Y. Quan, B. van den Brandt, J. Kohlbrecher, W. Th. Wenckebach, P. Hautle, Nucl. Instrum. Meth. Phys. Res. Sect. A 921, 22 (2019) 